\title{
Medicinal Plants Used In Traditional Treatment of Hypertension in Turkey
}

\author{
Bulent Olcay (Corresponding author) \\ Faculty of Pharmacy, Department of Pharmaceutical Botany, Istanbul University, \\ PO box 34116, Istanbul, Turkey. \\ E-mail: bulentolcay@istanbul.edu.tr \\ Sukran Kultur \\ Faculty of Pharmacy, Department of Pharmaceutical Botany, Istanbul University, \\ PO box 34116, Istanbul, Turkey. \\ E-mail: s_kultur@istanbul.edu.tr
}

\begin{abstract}
Since ancient times people have used plants because of their therapeutic effects. People have recorded the therapeutic effects of plants throughout history. Turkey has a rich traditional medicine and ethnobotanical culture. People have preserved this ethnobotanical culture by transferring them from generation to generation. Medicinal plants are playing an important role in health care programmes worldwide, especially in developing countries. In Turkey there are many plant species known with their blood pressure lowering effect. In this study 150 plant taxa, used against hypertension in Turkey were compiled. The study contains botanical names, families, local names, used parts and method of use of these plant species. According to this research commonly used medicinal plants are from Rosaceae (27 taxa), Lamiaceae (26 taxa), Asteraceae (15 taxa), Apiaceae (11 taxa), Polygonaceae (6 taxa), Loranthaceae (3 taxa) and Rhamnaceae (3 taxa) respectively.
\end{abstract}

Keywords: Hypertension, Traditional medicine, Ethnobotany, Medicinal plants, Turkey

\section{Special Issue of Health Sciences}

DOI: $10.7176 /$ JSTR/6-03-11

\section{Introduction}

Hypertension, also known as high or raised blood pressure, is a condition in which the blood vessels have persistently raised pressure (WHO). Optimal blood pressure is defined as a systolic blood pressure less than $120 \mathrm{~mm} \mathrm{Hg}$ and a diastolic blood pressure less than $80 \mathrm{~mm} \mathrm{Hg}$. Hypertension is characterized by a confirmed elevation of systolic $(\geq 140 \mathrm{~mm} \mathrm{Hg}$ ) or diastolic $(\geq 90 \mathrm{~mm} \mathrm{Hg}$ ) blood pressure (He and Whelton, 1997). Hypertension increases the risk of heart attacks, strokes and kidney failure. Uncontrolled hypertension can also cause blindness, irregular heartbeat and heart failure. Based on office blood pressure, the global prevalence of hypertension was estimated to be 1.13 billion in 2015 , with a prevalence of over 150 million in central and eastern Europe. The overall prevalence of hypertension in adults is around 30- 45\%, with a global age standardized prevalence of 24 and $20 \%$ in men and women, respectively, in 2015. This high prevalence of hypertension is consistent across the world, irrespective of income status, i.e. in lower, middle, and higher income countries. Hypertension becomes progressively more common with advancing age, with a prevalence of $>60 \%$ in people aged $>60$ years (Williams et al., 2018).

Turkey has a rich plant biodiversity and has ratio of endemism. Approximately twelve thousand plant species are growing in Turkey, and three thousand of them are endemic. (Güner et al., 2000, Özhatay et al. 2013, Özhatay et al., 2015, Özhatay et al., 2017) This rich plant biodiversity ensures that traditional medicine is widespread in Turkey. In the present study 151 plant taxa, used against hypertension in Turkey were obtained. The botanical names, families, local names, used parts and usage of these plant taxa are shown in Table 1. 
International Journal of Scientific and Technological Research

www.iiste.org

ISSN 2422-8702 (Online), DOI: 10.7176/JSTR/6-03-11

Special Issue of Health Sciences, Vol.6, No.3, 2020

Table 1- List of the medicinal plants used in traditional treatment of hypertension in Turkey.

\begin{tabular}{|c|c|c|c|c|c|}
\hline Botanical name & Family & Local name & $\begin{array}{l}\text { Plant part } \\
\text { used }\end{array}$ & $\begin{array}{l}\text { Preparation, } \\
\text { administration and use }\end{array}$ & References \\
\hline Achillea biebersteinii Afan. & Asteraceae & $\begin{array}{l}\text { Ayvadana, } \\
\text { Civamperçemi }\end{array}$ & Aerial parts & Inf. & (Tuzlacı 2016) \\
\hline $\begin{array}{l}\text { Achillea millefolium L. subsp. } \\
\text { pannonica (Schelek) Hayek }\end{array}$ & Asteraceae & $\begin{array}{l}\text { Civanperçemi, kurpotu, } \\
\text { dişotu, ayvadana, } \\
\text { ronağvaç, sporiş, }\end{array}$ & Flowers & $\begin{array}{l}\text { Inf., O.Ad., drink one } \\
\text { teacup two times a day } \\
\text { for } 5 \text { days }\end{array}$ & (Kültür 2007) \\
\hline Alchemilla sintenisii Rothm. & Rosaceae & $\begin{array}{l}\text { Aslanpençesi, } \\
\text { Fındık otu }\end{array}$ & Leaves & $\begin{array}{l}\text { Inf. One cup of plant in } \\
\text { the morning }\end{array}$ & (Tuzlacı 2016) \\
\hline $\begin{array}{l}\text { Alliaria petiolata } \\
\text { (M.Bieb.) Cavara \& Grande }\end{array}$ & Brassicaceae & Dida, Sarımsak otu & Aerial parts & Dec. & (Tuzlacı 2016) \\
\hline Allium cepa L. & Liliaceae & Soğan & Bulbs & $\begin{array}{l}\text { Inf., Drink one cup of the } \\
\text { plant on an empty } \\
\text { stomach in the morning }\end{array}$ & $\begin{array}{l}\text { (Hayta 2014; Saday 2009; Öztürk et al. } \\
\text { 2013; Tetik 2011; Tuzlacı 2016) }\end{array}$ \\
\hline $\begin{array}{l}\text { Allium macrochaetum Boiss. } \\
\text { et Hausskn. subsp. } \\
\text { tuncelianum Kollmann }\end{array}$ & Liliaceae & Dağ sarmısağı & Bulbs & Eaten & $\begin{array}{l}\text { (Tuzlacı \& Doğan 2010; Tuzlacı \& } \\
\text { Şenkardeş 2011; Doğan 2014; } \\
\text { Tuzlacı 2016) }\end{array}$ \\
\hline Allium sativum $\mathrm{L}$. & Liliaceae & Sarımsak, sarmısak & Bulbs, Dried & $\begin{array}{l}\text { O.Ad.; } \\
\text { Eaten }\end{array}$ & $\begin{array}{l}\text { (Polat et al. 2013; Güzel et al. 2015; } \\
\text { Karaman et al. 2001; Akbulut \& } \\
\text { Bayramoğlu 2013; } \\
\text { Han \& Bulut 2015; Polat \& Çakıroğlu } \\
\text { 2018; Sezik et al. 2001; Eşen 2008; } \\
\text { Tuzlacı \& Tolon 2000; Tuzlacı \& } \\
\text { Sadıkoğlu 2007; Ezer \& Arısan 2006; } \\
\text { Saday 2009; Bulut 2008; Çakılcığlu } \\
\text { et al. 2010; Tekin 2011; Onar 2006; } \\
\text { Metin 2009; Öztürk 2006; Şenkardeş } \\
\text { 2014; Tütenocaklı 2014; Polat 2010; } \\
\text { Kültür 2007; Tuzlacı 2016; Ezer \& } \\
\text { Arısan 2006) }\end{array}$ \\
\hline $\begin{array}{l}\text { Allium scorodoprassum } \mathrm{L} . \\
\text { subsp. rotundum }(\mathrm{L} \text {.) Stearn }\end{array}$ & Liliaceae & İt soğanı & Bulbs & $\begin{array}{l}\text { Fresh, dried. } \\
\text { Dec., Int. }\end{array}$ & $\begin{array}{l}\text { (Altundağ \& Ozturk 2011; Hayta 2014; } \\
\text { Altundağ 2009; Tuzlacı 2016) }\end{array}$ \\
\hline Allium tuncelianum & Liliaceae & Soğan & Bulbs & Fresh & (Ekşi 2012) \\
\hline Anchusa azurea Mill. & Boraginaceae & Gelazun, Gelezun & $\begin{array}{l}\text { Aerial parts, } \\
\text { Roots }\end{array}$ & $\begin{array}{l}\text { Dec., Aerial parts boiled. } \\
\text { Drink one tea glass of the } \\
\text { plant before the meal. } \\
\text { Eaten as meal }\end{array}$ & $\begin{array}{l}\text { (Polat et al. 2013; Akgul et al. 2018; } \\
\text { Tuzlacı 2016) }\end{array}$ \\
\hline Anethum graveolens L. & Apiaceae & Dereotu & Seeds & $\begin{array}{l}\text { Dec., } 0 . \text { Ad, drink one } \\
\text { teacup three times a day } \\
\text { for } 7-8 \text { days }\end{array}$ & $\begin{array}{l}\text { (Ugulu et al. 2009; Kaval et al. 2014; } \\
\text { Bulut et al. 2008; Tuzlacı 2016) }\end{array}$ \\
\hline $\begin{array}{l}\text { Anthemis coelopoda var. } \\
\text { bourgaei Boiss. }\end{array}$ & Asteraceae & Papatya & Aerial parts & $\begin{array}{l}\text { Inf., Drink one cup of the } \\
\text { plant on an empty } \\
\text { stomach in the morning }\end{array}$ & $\begin{array}{l}\text { (Tetik et al. 2013; Tetik 2011; Tuzlacı } \\
\text { 2016) }\end{array}$ \\
\hline $\begin{array}{l}\text { Anthemis tinctoria L. var. } \\
\text { tinctoria }\end{array}$ & Asteraceae & $\begin{array}{l}\text { Papatya, } \\
\text { Sarı papatya }\end{array}$ & Flowers & Dec. & (Doğan 2014; Tuzlacı 2016) \\
\hline Apium nodiflorum (L.) Lag. & Apiaceae & Bendik & Aerial parts & Eaten & $\begin{array}{l}\text { (Bulut et al. 2008; Demirci 2010; } \\
\text { Tuzlacı 2016) }\end{array}$ \\
\hline Arbutus unedo L. & Ericaceae & $\begin{array}{l}\text { Kocayemiş, Dağ } \\
\text { yemişi }\end{array}$ & Fruits & Eaten & (Kızılarslan 2008) \\
\hline Artedia squamata L. & Apiaceae & Karabenek & Leaves & Inf., Int. & $\begin{array}{l}\text { (Bulut et al. 2008; Doğan 2014; } \\
\text { Tuzlacı 2016) }\end{array}$ \\
\hline Artemisia absinthium L. & Asteraceae & $\begin{array}{l}\text { Pelinotu, acıpelin, } \\
\text { pelin, acı pelinotu }\end{array}$ & Leaves & $\begin{array}{l}\text { Cutting into little pieces/ } \\
\text { Ext., chewed once a day } \\
\text { for } 10 \text { days. } \\
\text { Dec., O.Ad., drink one } \\
\text { teacup once a day for } 5 \\
\text { days }\end{array}$ & $\begin{array}{l}\text { (Özhatay et al. 2006; Kültür 2007; } \\
\text { Tuzlacı 2016) }\end{array}$ \\
\hline $\begin{array}{l}\text { Arum elongatum Steven } \\
\text { subsp. detruncatum (C.A. } \\
\text { Meyer ex Schott) H. Riedl. }\end{array}$ & Araceae & $\begin{array}{l}\text { Kardun, Kardu, } \\
\text { Kardı }\end{array}$ & Leaves & $\begin{array}{l}\text { Int., Compress Drink one } \\
\text { cup of the plant on an } \\
\text { empty stomach in the } \\
\text { morning }\end{array}$ & $\begin{array}{l}\text { (Polat et al. 2013; Polat \& Çakıroğlu } \\
\text { 2018; Tuzlacı 2016) }\end{array}$ \\
\hline
\end{tabular}


International Journal of Scientific and Technological Research

ISSN 2422-8702 (Online), DOI: 10.7176/JSTR/6-03-11

Special Issue of Health Sciences, Vol.6, No.3, 2020

\begin{tabular}{|c|c|c|c|c|c|}
\hline Botanical name & Family & Local name & $\begin{array}{l}\text { Plant part } \\
\text { used }\end{array}$ & $\begin{array}{l}\text { Preparation, } \\
\text { administration and use }\end{array}$ & References \\
\hline Ballota nigra L. subsp. nigra & Lamiaceae & Arıotu & $\begin{array}{l}\text { Flowers, } \\
\text { Leaves }\end{array}$ & Inf. & (Tetik et al. 2013; Tuzlacı 2016) \\
\hline Bellis perennis $\mathrm{L}$. & Asteraceae & Beyazpapatya & $\begin{array}{l}\text { Flowers, } \\
\text { Leaves }\end{array}$ & Inf., Dec. & (Kızılarslan 2008) \\
\hline Berberis vulgaris L. & Berberidaceae & $\begin{array}{l}\text { Kadın tuzluğu, } \\
\text { Karamuk }\end{array}$ & $\begin{array}{l}\text { Root, } \\
\text { Leaves, } \\
\text { Flower, Fruit }\end{array}$ & Crude, dried and Dec. & (Akgül et al. 2016) \\
\hline Berberis crataegina DC. & Berberidaceae & Karamık & Fruits & The plant is eaten raw & $\begin{array}{l}\text { (Eşen 2008; Saday 2009; Tuzlacı } \\
2016)\end{array}$ \\
\hline $\begin{array}{l}\text { Brassica nigra (L.) W.D.J. } \\
\text { Koch }\end{array}$ & Brassicaceae & Eşek turpu & Leaves, stem & Inf. & (Güneş 2010) \\
\hline $\begin{array}{l}\text { Bryonia multiflora Boiss. \& } \\
\text { Heldr. }\end{array}$ & Cucurbitaceae & Juri ruvi, tırye ruvi & Fruits & The plant is eaten raw & (Mükemre et al. 2015) \\
\hline Calendula officinalis $L$. & Asteraceae & Aynısafa & Aerial Parts & \begin{tabular}{|l|} 
Dec. 0. Ad, drink one \\
teacup $2-3$ times a day \\
for $5-6$ days
\end{tabular} & (Ugulu et al. 2009; Tuzlacı 2016) \\
\hline $\begin{array}{l}\text { Capsella bursa-pastoris } \\
\text { Medik. }\end{array}$ & Brassicaceae & Çoban çantası & Leaves & $\begin{array}{l}\text { Dec. O., drink one teacup } \\
3 \text { times a day for } 8 \text { days }\end{array}$ & (Ugulu et al. 2009) \\
\hline Castanea sativa Mill. & Fagaceae & Anadolu kestanesi & Cortex & Inf. & $\begin{array}{l}\text { (Saraç 2013; Kızılarslan 2008; } \\
\text { Tuzlacı 2016) }\end{array}$ \\
\hline Chaerophyllum bulbosum L. & Apiaceae & Handok & $\begin{array}{l}\text { Rhizome, } \\
\text { Leaves }\end{array}$ & Inf., Int. & (Bulut et al. 2008; Doğan 2014) \\
\hline Celtis australis $\mathrm{L}$. & Cannabaceae & Karaçıtlık, çitlenbik & Fruits, leaves & Dec., Int. & $\begin{array}{l}\text { (Tuzlacı \& Sadıkoğlu 2007; Tuzlacı } \\
\text { 2016) }\end{array}$ \\
\hline Ceratonia siliqua L. & Fabaceae & $\begin{array}{l}\text { Cırnıp, Harnup, } \\
\text { Keçiboynuzu }\end{array}$ & Fruits & Inf. & (Güneş 2010) \\
\hline Cichorium intybus L. & Asteraceae & Kaniş & $\begin{array}{l}\text { Aerial parts, } \\
\text { latex, whole } \\
\text { plant }\end{array}$ & $\begin{array}{l}\text { Inf., Eaten as meal. Drink } \\
\text { one glass } 2-3 \text { times a } \\
\text { day for } 8-12 \text { weeks }\end{array}$ & $\begin{array}{l}\text { (Kaval et al. 2014; Sargın et al. 2013; } \\
\text { Tetik et al. 2013; Mükemre et al. } \\
\text { 2015; Akgul et al. 2018; Tuzlacı 2016) }\end{array}$ \\
\hline Cistus laurifolius L. & Cistaceae & $\begin{array}{l}\text { Yavşancıl, tistüs, } \\
\text { karakan yaprağı, } \\
\text { karakan otu }\end{array}$ & Leaves & Dec., Inf. & (Oral 2007; Tuzlacı 2016) \\
\hline Citrus x limon & Rutaceae & Limon & Fruits & The fruits are eaten raw & (Eşen 2008) \\
\hline Cnicus benedictus L. & Asteraceae & $\begin{array}{l}\text { Devedikeni, } \\
\text { Şevketi bostan }\end{array}$ & Whole plant & $\begin{array}{l}\text { Int., Dec., spice Drink one } \\
\text { teacup } 2-3 \text { times a day } \\
\text { for 3-4 weeks }\end{array}$ & (Sargın et al. 2013) \\
\hline $\begin{array}{l}\text { Cnicus benedictus L. var. } \\
\text { benedictus }\end{array}$ & Asteraceae & Mübarek dikeni & Flowers, leaves & $\begin{array}{l}\text { Dec., drink one cup of the } \\
\text { plant on an empty } \\
\text { stomach in the morning }\end{array}$ & $\begin{array}{l}\text { (Cakilcioglu \& Turkoglu 2010; Tuzlacı } \\
\text { 2016) }\end{array}$ \\
\hline Cornus mas L. & Cornaceae & Kızılcık & Fruits & $\begin{array}{l}\text { Dec., O. Ad., drink one } \\
\text { tea cup two times a day } \\
\text { for a week }\end{array}$ & $\begin{array}{l}\text { (Polat et al. 2011; Güneş 2010; Polat } \\
\text { 2010; Tuzlacı 2016) }\end{array}$ \\
\hline Cotinus coggygria Scop. & Anacardiaceae & $\begin{array}{l}\text { Tetra, tetre, tetere, } \\
\text { tetra otu }\end{array}$ & Leaves & Dec., O.Ad. & (Kültür 2007; Tuzlacı 2016) \\
\hline $\begin{array}{l}\text { Crataegus aronia (L.) Bosc ex } \\
\text { DC. var. aronia (L.) Bosc ex } \\
\text { DC. }\end{array}$ & Rosaceae & $\begin{array}{l}\text { Zeğrur, } \\
\text { Alıç }\end{array}$ & Leaves, flowers & Dec. & $\begin{array}{l}\text { (Güzelşemme 2014; Gençay 2007; } \\
\text { Tuzlacı 2016) }\end{array}$ \\
\hline $\begin{array}{l}\text { Crataegus monogyna Jacq. } \\
\text { subsp. monogyna }\end{array}$ & Rosaceae & $\begin{array}{l}\text { Yemişgen, alıç, arıç, } \\
\text { yemişgen çalısı }\end{array}$ & Fruits, flowers & Dec., Int., 3,4 times a day & $\begin{array}{l}\text { (Koçyiğit et al. 2006; Bulut et al. 2013; } \\
\text { Fakir et al. 2009; Arı et al. 2015; } \\
\text { Karaman et al. 2001; } \\
\text { Eşen 2008; Saday 2009; Doğan } \\
\text { 2014; Bulut 2008; Güzelşemme 2014; } \\
\text { Tekin 2011; Metin 2009; Şahin 2014; } \\
\text { Alparslan 2012; Güldaş 2009; } \\
\text { Şenkardeş; Savran et al. 2009; Genç } \\
\text { 2003; Genç 2006; } \\
\text { Tuzlacı 2016) }\end{array}$ \\
\hline $\begin{array}{l}\text { Crataegus pentagyna Waldst. } \\
\text { et Kit. ex Willd }\end{array}$ & Rosaceae & $\begin{array}{l}\text { Alıç, } \\
\text { Alişan çalısı }\end{array}$ & \begin{tabular}{|l} 
Flowers, \\
Leaves
\end{tabular} & As cardiotonic; Inf. & $\begin{array}{l}\text { (Koçyiğit et al. 2006, Arı et al. 2015; } \\
\text { Tuzlacı 2016) }\end{array}$ \\
\hline
\end{tabular}


International Journal of Scientific and Technological Research

ISSN 2422-8702 (Online), DOI: 10.7176/JSTR/6-03-11

Special Issue of Health Sciences, Vol.6, No.3, 2020

\begin{tabular}{|c|c|c|c|c|c|}
\hline Botanical name & Family & Local name & $\begin{array}{l}\text { Plant part } \\
\text { used }\end{array}$ & \begin{tabular}{|l|} 
Preparation, \\
administration and use
\end{tabular} & References \\
\hline Crataegus pontica C. KOCH & Rosaceae & $\begin{array}{l}\text { Zeğrur, Alıç, Masmus, } \\
\text { Kırmızı alıç }\end{array}$ & $\begin{array}{l}\text { Fruits, } \\
\text { leaves, } \\
\text { flowers }\end{array}$ & Dec. & (Güzelşemme 2014) \\
\hline $\begin{array}{l}\text { Crataegus } \\
\text { pseudoheterophylla Pojark. }\end{array}$ & Rosaceae & Alıç, Aluç & $\begin{array}{l}\text { Flower, } \\
\text { leaves }\end{array}$ & Inf. & (Doğan 2014; Tuzlacı 2016) \\
\hline Crataegus tanacetifolia (Lam.) & Rosaceae & Alıç & Flowers & $\begin{array}{l}\text { Inf., Int., drink one glass } \\
\text { once a day }\end{array}$ & (Uzun \& Kaya 2016; Mumcu 2010) \\
\hline $\begin{array}{l}\text { Crataegus } x \text { bornmuelleri } \\
\text { Zabel }\end{array}$ & Rosaceae & Gıvica zar & Roots & Inf. & (Yeşil \& Akalın 2009) \\
\hline $\begin{array}{l}\text { Convolvulus betonicifolius Mill. } \\
\text { subsp. peduncularis (Boiss.) } \\
\text { Paris }\end{array}$ & Convolvulaceae & Sarmaşık & Aerial parts, & $\begin{array}{l}\text { Inf., drink one cup of the } \\
\text { plant on an empty } \\
\text { stomach in the morning }\end{array}$ & (Hayta 2014) \\
\hline \begin{tabular}{l|l} 
Cydonia oblonga Miller & \\
\end{tabular} & Rosaceae & Ayva & Leaves & Int. & (Bulut et al. 2013; Tuzlacı 2016) \\
\hline $\begin{array}{l}\text { Dioscorea communis (L.) } \\
\text { Caddick \& Wilkin }\end{array}$ & Dioscoreaceae & $\begin{array}{l}\text { Acı ot, vicirne, } \\
\text { kabarcık, kapırcık }\end{array}$ & $\begin{array}{l}\text { Roots, flower } \\
\text { bark of mature } \\
\text { fruit }\end{array}$ & $\begin{array}{l}\text { Raw(uncooked), Aerial } \\
\text { parts boiled. }\end{array}$ & (Sargın et al. 2013) \\
\hline Diplotaenia cachrydifolia Boiss & Apiaceae & Siyabu & \begin{tabular}{|l|} 
Aerial parts, \\
roots
\end{tabular} & $\begin{array}{l}\text { Dec., The plant is eaten } \\
\text { raw }\end{array}$ & (Mükemre et al. 2015) \\
\hline $\begin{array}{l}\text { Dryopteris pallida (Bory) } \\
\text { Fomin. }\end{array}$ & Dryopteridaceae & Eğrelti otu & Aerial parts & Dec. & (Oral 2007; Tuzlacı 2016) \\
\hline Equisetum arvense $\mathrm{L}$. & Equisetaceae & $\begin{array}{l}\text { Giyagezık, } \\
\text { Atkuyruğu }\end{array}$ & Aerial parts & \begin{tabular}{|l|} 
Inf., Drink one glass of \\
the plant on an empty \\
stomach in the morning. \\
Drink one glass 3 times a \\
day for $4-8$ weeks \\
\end{tabular} & $\begin{array}{l}\text { (Kaval et al. 2014; Sargın et al. 2013; } \\
\text { Güzel et al. 2015) }\end{array}$ \\
\hline Erica arborea L. & Ericaceae & Süpürge otu & Aerial parts & - & (Kızılarslan 2008) \\
\hline Erica manipuliflora Salisb. & Ericaceae & $\begin{array}{l}\text { Funda, } \\
\text { Püren }\end{array}$ & $\begin{array}{l}\text { Flowers and } \\
\text { leaves }\end{array}$ & Dec., Int. & (Tuzlacı \& Sadıkoğlu 2007) \\
\hline Fumaria officinalis L. & Fumariaceae & Şahtere otu & Aerial parts & $\begin{array}{l}\text { Inf. } 0 . \text { Ad. Drink one } \\
\text { teacup two times a day } \\
\text { for } 1-2 \text { weeks }\end{array}$ & $\begin{array}{l}\text { (Polat \& Satıl 2011; Oral 2007; Polat } \\
\text { 2010; Tuzlacı 2016) }\end{array}$ \\
\hline Geum urbanum L. & Rosaceae & $\begin{array}{l}\text { Bit otu, } \\
\text { Yellice otu }\end{array}$ & Aerial parts & Inf. & (Doğan 2014; Tuzlacı 2016) \\
\hline Glycyrrhyza glabra L. & Fabaceae & Meyan kökü & Roots & Inf. & (Akan et al. 2013) \\
\hline Helianthus tuberosus L. & Asteraceae & $\begin{array}{l}\text { Say erd, Say bin erd, } \\
\text { Yer elması }\end{array}$ & Tuber & The plant is eaten raw & (Polat et al. 2013) \\
\hline $\begin{array}{l}\text { Helichrysum compactum } \\
\text { Boiss. }\end{array}$ & Asteraceae & Altınotu & Leaves & $\begin{array}{l}\text { Dec. ( } 2-3 \text { cups of tea } \\
\text { daily) }\end{array}$ & (Kargıoğlu et al. 2010; Vural 2008) \\
\hline Hibiscus esculentus L. & Malvaceae & Bamya & Flowers & - & (Uysal 2010) \\
\hline Hibiscus sabdariffa L. & Malvacaceae & $\begin{array}{l}\text { Habiskus, } \\
\text { Medine gülü }\end{array}$ & Calyx & Inf. & (Akan 2015) \\
\hline Hypericum perforatum L. & Hypericaceae & $\begin{array}{l}\text { Kantaron, kantaron } \\
\text { çayı, sarı kantaron, } \\
\text { kantaryon, sarıcayüz, } \\
\text { kantül, kesik otu, mide } \\
\text { otu, kalp otu }\end{array}$ & Aerial parts & Dec., O.Ad. & $\begin{array}{l}\text { (Akaydın et al. 2013; Bulut \& Tuzlacı } \\
\text { 2013; Özhatay et al. 2006; Kültür } \\
\text { 2007; } \\
\text { Tuzlacı 2016) }\end{array}$ \\
\hline Juglans regia L. & Juglandaceae & Ceviz & Leaves & $\begin{array}{l}\text { Dec., Int., drink one glass } \\
\text { once a day }\end{array}$ & $\begin{array}{l}\text { (Uzun \& Kaya 2016; Kızılarslan 2008; } \\
\text { Metin 2009; Tuzlacı 2016) }\end{array}$ \\
\hline Juniperus foetidissima Willd. & Cupressaceae & Ardıç & Resins & \begin{tabular}{|l|} 
Dec, resins chewed, \\
Apply chewed gum $2-3$ \\
times a day for $1-2$ \\
weeks /chew $1 / 2$ handful \\
aday for $2-3$ weeks.
\end{tabular} & $\begin{array}{l}\text { (Hayta 2014; Sargın et al. 2015; Bulut } \\
\text { 2008; Tuzlacı 2016) }\end{array}$ \\
\hline Juniperus oxycedrus L. & Cupressaceae & $\begin{array}{l}\text { Pardı üzümü, } \\
\text { Andız meyvesi }\end{array}$ & Fruits & Dec, Inf. & (Oral 2007; Tuzlacı 2016) \\
\hline Lactuca saligna L. & Asteraceae & Tehliska geva & Flowers & The plant is eaten raw & (Kaval et al. 2014; Tuzlacı 2016) \\
\hline $\begin{array}{l}\text { Laurocerasus officinalis } \\
\text { Roemer }\end{array}$ & Rosaceae & $\begin{array}{l}\text { Karayemiş, } \\
\text { Taflan }\end{array}$ & Leaves & The plant is eaten raw & (Polat et al. 2015) \\
\hline
\end{tabular}


International Journal of Scientific and Technological Research

ISSN 2422-8702 (Online), DOI: 10.7176/JSTR/6-03-11

Special Issue of Health Sciences, Vol.6, No.3, 2020

\begin{tabular}{|c|c|c|c|c|c|}
\hline Botanical name & Family & Local name & $\begin{array}{l}\text { Plant part } \\
\text { used }\end{array}$ & \begin{tabular}{|l|} 
Preparation, \\
administration and use
\end{tabular} & References \\
\hline $\begin{array}{l}\text { Lavandula stoechas L. subsp. } \\
\text { stoechas }\end{array}$ & Lamiaceae & Karabaş otu, Karabaş & $\begin{array}{l}\text { Flowering } \\
\text { branches }\end{array}$ & $\begin{array}{l}\text { Inf. } 0 . \text { Ad. drink one } \\
\text { teacup two times a day } \\
\text { for } 1-2 \text { weeks }\end{array}$ & $\begin{array}{l}\text { (Polat \& Satıl 2011; Sargın 2015; } \\
\text { Güzel et al. 2015; Uysal et al. 2012; } \\
\text { Tuzlacı \& Sadıkoğlu 2007; } \\
\text { Güzelşemme 2014; Onar 2006; } \\
\text { Tuzlacı 2016) }\end{array}$ \\
\hline Laser trilobum (L.) Borkh & Apiaceae & Siraotu & Fruits & $\begin{array}{l}\text { Eaten raw or cooked, } \\
\text { Dec. }\end{array}$ & (Güneş et al. 2017; Güneş 2010) \\
\hline Lecokia cretica (Lam.) DC. & Apiaceae & Baldıran & Seeds & Crushed. Int & $\begin{array}{l}\text { (Bulut et al. 2008; Tuzlacı \& } \\
\text { Sadıkoğlu 2007; Demirci \& Özhatay } \\
\text { 2012; Tuzlacı 2016) }\end{array}$ \\
\hline $\begin{array}{l}\text { Linum aroanium Boiss. \& } \\
\text { Orph. }\end{array}$ & Linaceae & Keten & Seeds & $\begin{array}{l}\text { Grinded seeds/mixed } \\
\text { with yogurt and eaten } \\
\text { Roasted and grinded } \\
\text { seeds/boiled and drunk } \\
\text { as coffee }\end{array}$ & $\begin{array}{l}\text { (Güzel et al. 2015; Güzelşemme } \\
\text { 2014) }\end{array}$ \\
\hline $\begin{array}{l}\text { Linum hirsutum L. subsp. } \\
\text { pseudoanatolicum P.H. Davis }\end{array}$ & Linaceae & $\begin{array}{l}\text { Siyerek, } \\
\text { Keten }\end{array}$ & Seeds & Crushed seeds are eaten & (Deniz 2008; Tuzlacı 2016) \\
\hline Linum usitatissimum L. & Linaceae & Keten & Seeds & Dec. & (Güzelşemme 2014; Akan 2015) \\
\hline $\begin{array}{l}\text { Lippia trphylla (L'Her.) } 0 . \\
\text { Kuntze }\end{array}$ & Verbenaceae & $\begin{array}{l}\text { Limon otu, Limonlu } \\
\text { melisa, Ağaç lavanta }\end{array}$ & $\begin{array}{l}\text { Leaves, } \\
\text { flowers }\end{array}$ & Dec. & (Güzelşemme 2014) \\
\hline $\begin{array}{l}\text { Malus sylvestris Miller subsp. } \\
\text { mitis (Wallr.) Mansf. }\end{array}$ & Rosaceae & $\begin{array}{l}\text { Bodur elma, Taar } \\
\text { elması }\end{array}$ & Fruits & Eaten, $2-3$ fruits $x 1$ & $\begin{array}{l}\text { (Tuzlacı \& Şenkardeş 2011; } \\
\text { Şenkardeş 2014; Tuzlacı 2016) }\end{array}$ \\
\hline $\begin{array}{l}\text { Malus sylvestris Miller subsp. } \\
\text { orientalis (A. Uglitzkich) } \\
\text { Browicz var. orientalis }\end{array}$ & Rosaceae & $\begin{array}{l}\text { Sev, } \\
\text { Yabani elma }\end{array}$ & Fruits & Fruits are eaten raw & (Doğan 2014) \\
\hline Malva neglecta Wallr. & Malvaceae & $\begin{array}{l}\text { Gömeç, } \\
\text { Ebegümeci }\end{array}$ & $\begin{array}{l}\text { Roots, } \\
\text { stem }\end{array}$ & Dec. & $\begin{array}{l}\text { (Güneş 2010; Demirci 2010; Tuzlacı } \\
\text { 2016) }\end{array}$ \\
\hline Malva sylvestris L. & Malvaceae & $\begin{array}{l}\text { Gömeç, } \\
\text { Ebegümeci }\end{array}$ & \begin{tabular}{|l|l}
$\begin{array}{l}\text { Roots, } \\
\text { stem }\end{array}$ \\
\end{tabular} & Dec. & (Güneş 2010; Tuzlacı 2016) \\
\hline Melisa officinalis L. & Lamiaceae & Melisa, oğulotu & Leaves, flowers & Dec. & $\begin{array}{l}\text { (Uysal et al. 2010; Oral 2007;Onar } \\
2006)\end{array}$ \\
\hline $\begin{array}{l}\text { Melissa officinalis L. subsp. } \\
\text { altissima (Sm.) Arcangeli }\end{array}$ & Lamiaceae & Oğulotu, Saçkıran & Aerial parts, & Dec. & (Kızılarslan 2008) \\
\hline Mentha pulegium L. & Lamiaceae & Narpuz, yarpuz & Aromatic water & Int. & (Gürdal \& Kültür 2013) \\
\hline $\begin{array}{l}\text { Mentha spicata L. subsp. } \\
\text { tomentosa (Briq.) Harley }\end{array}$ & Lamiaceae & Yarpuz & Leaves & Inf. & (Erdoğan 2011) \\
\hline Mespilus germanica L. & Rosaceae & Döngel, Muşmula & \begin{tabular}{|l|} 
Fruits, \\
leaves
\end{tabular} & $\begin{array}{l}\text { The plant is eaten raw, } \\
\text { Dec. }\end{array}$ & $\begin{array}{l}\text { (Polat et al. 2015; Tuzlacı \& Alparslan } \\
\text { 2013; Kızılarslan 2008; Tuzlacı 2016) }\end{array}$ \\
\hline Musa acuminata Colla & Musaceae & Muz & Fruits & $\begin{array}{l}\text { Fruits are eaten by } \\
\text { chewing. Eat } 3-4 \text { pieces a } \\
\text { day for } 2-3 \text { weeks. }\end{array}$ & $($ Sargın 2015) \\
\hline Nasturtium officinale $\mathrm{R} . \mathrm{Br}$ & Brassicaceae & Kijı, Qije, Tujik & Aerial parts & $\begin{array}{l}\text { Int., Drink one tea glass } \\
\text { of the plant before the } \\
\text { meal }\end{array}$ & $\begin{array}{l}\text { (Polat et al. 2013; Polat \& Çakıroğlu } \\
\text { 2018; Demirci 2010; Tuzlacı 2016) }\end{array}$ \\
\hline $\begin{array}{l}\text { Olea europaea L. var. } \\
\text { europaea }\end{array}$ & Oleaceae & Zeytin & Leaves & Inf., Int. & $\begin{array}{l}\text { (Bulut \& Tuzlacı 2013; Sargın et al. } \\
\text { 2013; Sargın 2015; Güzel et al. 2015; } \\
\text { Sağıroğlu et al. 2013; Gürdal \& Kültür } \\
\text { 2013; Bulut 2008; Onar 2006; Metin } \\
\text { 2009; Tuzlacı 2016) }\end{array}$ \\
\hline $\begin{array}{l}\text { Olea europaea L. var. } \\
\text { sylvestris (Miller) Lehr. }\end{array}$ & Oleaceae & Yabani zeytin & Leaves & Inf. & $\begin{array}{l}\text { (Fakir et al. 2009; Öztürk et al. 2013; } \\
\text { Tuzlacı 2016) }\end{array}$ \\
\hline Origanum onites L. & Lamiaceae & $\begin{array}{l}\text { Kırkbaş kekik, Tokalı } \\
\text { kekik, Arı kekiği }\end{array}$ & Aerial parts & Inf, Aerial parts crushed & $\begin{array}{l}\text { (Sargın et al. 2013; Gürdal \& Kültür } \\
\text { 2013; Tuzlacı \& Erol 1999; Tuzlacı \& } \\
\text { Sadıkoğlu 2007; } \\
\text { Tuzlacı 2016) }\end{array}$ \\
\hline Origanum vulgare $\mathrm{L}$. & Lamiaceae & Kekik & Leaves & $\begin{array}{l}\text { Dec., Drink one tea glass } \\
\text { of the plant two times a } \\
\text { day }\end{array}$ & (Hayta 2014) \\
\hline $\begin{array}{l}\text { Origanum vulgare L. subsp. } \\
\text { gracile (C. Koch) letswaart }\end{array}$ & Lamiaceae & Eşek kekiği & Aerial parts & Dec., Inf., Int. & $\begin{array}{l}\text { (Altundağ \& Ozturk 2011; Altundağ } \\
\text { 2009; Tuzlacı 2016) }\end{array}$ \\
\hline Paliurus spina-christi Miller & Rhamnaceae & Karaçalı, Dikenli çalı & $\begin{array}{l}\text { Fruits, } \\
\text { leaves, aerial } \\
\text { parts }\end{array}$ & Inf. & (Kızılarslan 2008; Tuzlacı 2016) \\
\hline Papaver bracteatum Lindl & Papaveraceae & Haşhaş & Seeds & The seeds are eaten raw. & (Mükemre et al. 2015) \\
\hline Peganum harmala L. & Zygophyllaceae & Üzerlik & Seeds & Dec., Inf. & (Oral 2007; Tuzlacı 2016) \\
\hline
\end{tabular}


International Journal of Scientific and Technological Research

ISSN 2422-8702 (Online), DOI: 10.7176/JSTR/6-03-11

Special Issue of Health Sciences, Vol.6, No.3, 2020

\begin{tabular}{|c|c|c|c|c|c|}
\hline Botanical name & Family & Local name & $\begin{array}{l}\text { Plant part } \\
\text { used }\end{array}$ & \begin{tabular}{|l|} 
Preparation, \\
administration and use
\end{tabular} & References \\
\hline $\begin{array}{l}\text { Petroselinum crispum (Miller) } \\
\text { A. W. Hill }\end{array}$ & Apiaceae & Maydanoz & $\begin{array}{l}\text { Leaves and } \\
\text { petioles }\end{array}$ & $\begin{array}{l}\text { Inf. O.Ad., } \\
\text { Dec. }\end{array}$ & $\begin{array}{l}\text { (Güzel et al. 2015; Karaman et al. } \\
\text { 2001; Güler et al. 2013; Uzun \& Kaya } \\
\text { 2016; Bulut et al. 2008; } \\
\text { Yazıcıoğlu \& Tuzlacı 1995; Tuzlacı } \\
\text { 2016) }\end{array}$ \\
\hline Physalis alkekengi $\mathrm{L}$. & Solanaceae & $\begin{array}{l}\text { Güney feneri, } \\
\text { Patlangaç, } \\
\text { Fener çiçeği }\end{array}$ & Fruits & Eaten & (Saraç 2013; Tuzlacı 2016) \\
\hline Plantago major L. & Plantaginaceae & Damar otu & Leaves & Inf. & (Akbulut \& Bayramoğlu 2013) \\
\hline $\begin{array}{l}\text { Plantago major L. subsp. } \\
\text { major }\end{array}$ & Plantaginaceae & $\begin{array}{l}\text { Balazagva, Damarliot } \\
\text { Damarotu, Kirksinirotu }\end{array}$ & Leaves & $\begin{array}{l}\text { Dec., Int., before } \\
\text { breakfast }\end{array}$ & $\begin{array}{l}\text { (Kızılarslan \& Özhatay 2012; } \\
\text { Kızılarslan 2008; Tuzlacı 2016) }\end{array}$ \\
\hline Portulaca oleracea L. & Portulaceae & Semiz otu & Aerial parts & The plant is eaten raw & (Eşen 2008) \\
\hline Prangos ferulacea (L.) Lindl. & Apiaceae & Eşek çakşırı & Leaves & Inf., Internal & $\begin{array}{l}\text { (Bulut et al. 2008; Doğan 2014; } \\
\text { Özgen et al. 2012) }\end{array}$ \\
\hline Prunus cerasus L. & Rosaceae & Vişne & Fruits & Eaten raw & (Tekin 2011; Karagöz 2013;) \\
\hline Prunus cocomilia Ten. & Rosaceae & Ekşi erik, Dağ eriği & Fruits & Fresh eaten & (Simsek 2004) \\
\hline Prunus divaricata Ledeb. & Rosaceae & Deli erik & Fruits & Fresh O. Ad. eaten & Polat \& Satıl 2011 \\
\hline $\begin{array}{l}\text { Prunus divaricata Ledeb. } \\
\text { subsp. divaricata }\end{array}$ & Rosaceae & $\begin{array}{l}\text { Deli erik, Yabani erik, } \\
\text { dağ eriği }\end{array}$ & Fruits & $\begin{array}{l}\text { Fruits are crashed, dried, } \\
\text { maserated, filtered, raw }\end{array}$ & (Sargın et al. 2015; Sargın 2015) \\
\hline Punica granatum $\mathrm{L}$. & Punicaceae & Nar & $\begin{array}{l}\text { Flowers } \\
\text { Fruits }\end{array}$ & \begin{tabular}{|l|} 
Dec. \\
Fresh eaten
\end{tabular} & $\begin{array}{l}\text { (Uysal et al. 2010; Kılıç \& Bağcı 2013; } \\
\text { Uzun \& Kaya 2016; Güneş 2010; } \\
\text { Güzelşemme 2014; Öztürk et al. } \\
\text { 2013; Onar 2006; Akan 2015; Tuzlacı } \\
\text { 2016) }\end{array}$ \\
\hline $\begin{array}{l}\text { Pyrus elaeagnifolia Pallas } \\
\text { subsp. elaeagnifolia Pallas }\end{array}$ & Rosaceae & Ahlat & Fruit & $\begin{array}{l}\text { Inf. as tea(4 cups a day) } \\
\text { Fruits of the plant part are } \\
\text { eaten directly. }\end{array}$ & (Arı et al. 2015) \\
\hline Quercus brantii Lindley & Fagaceae & $\begin{array}{l}\text { Meşe, } \\
\text { Palamut, Çılo }\end{array}$ & Fruits & Eaten raw & (Gençay 2007) \\
\hline $\begin{array}{l}\text { Quercus ithaburensis Decne } \\
\text { subsp. macrolepis (Kotschy) } \\
\text { Hedge \& Yalt }\end{array}$ & Fagaceae & $\begin{array}{l}\text { Palamut, } \\
\text { Pilemit }\end{array}$ & Fruits & Eaten raw & (Gençay 2007) \\
\hline Rheum ribes $\mathrm{L}$. & Polygonaceae & Işgın & Root, shoots & Dec, Int. & $\begin{array}{l}\text { (Altundağ \& Ozturk 2011; Korkmaz \& } \\
\text { Karakuş 2015; Altundağ 2009; Tekin } \\
\text { 2011; Gençay 2007; } \\
\text { Tuzlacı 2016) } \\
\end{array}$ \\
\hline Rhus coriaria L. & Anacardiaceae & Sumak & Matured fruits & $\begin{array}{l}\text { Dec., Inf. Drink one cup } \\
\text { of the plant on an empty } \\
\text { stomach in the morning }\end{array}$ & $\begin{array}{l}\text { (Polat et al. 2015; Hayta 2014; Polat } \\
\text { et al. 2013; Polat \& Çakıroğlu 2018) }\end{array}$ \\
\hline Rosa canina L. & Rosaceae & Kuşburnu & Fruits & Dec., Int. & $\begin{array}{l}\text { (Güler et al. 2013; Tuzlacı \& Erol } \\
\text { 1999; Şahin 2014; Savran et al. 2009; } \\
\text { Akan 2015; } \\
\text { Mumcu 2010;101) }\end{array}$ \\
\hline Rosmarinus officinalis L. & Lamiaceae & Kuş dili & Aerial parts & $\begin{array}{l}\text { Dec., O.Ad., drink one } \\
\text { teacup two times a day } \\
\text { for } 5-10 \text { days }\end{array}$ & $\begin{array}{l}\text { (Özhatay et al. 2006; Güneş 2010; } \\
\text { Kızılarslan 2008; Onar 2006; } \\
\text { Metin 2009; Kültür 2007; Tuzlacı } \\
\text { 2016) }\end{array}$ \\
\hline $\begin{array}{l}\text { Rubus canescens DC. var. } \\
\text { canescens }\end{array}$ & Rosaceae & $\begin{array}{l}\text { Böğürtlen, } \\
\text { karamuk }\end{array}$ & $\begin{array}{l}\text { Roots, fruits, } \\
\text { aerial parts }\end{array}$ & Dec. & (Kızılarslan 2008; Tuzlacı 2016) \\
\hline $\begin{array}{l}\text { Rubus canescens DC. var. } \\
\text { glabrescens (Godron) Davis \& } \\
\text { Meikle }\end{array}$ & Rosaceae & Böğürtlen & $\begin{array}{l}\text { Shoots, roots, } \\
\text { fruit }\end{array}$ & Dec., Int & (Altundağ \& Ozturk 2011) \\
\hline \begin{tabular}{l|} 
Rubus sanctus Schreb. \\
\end{tabular} & Rosaceae & Böğürtlen & Fruits & Eaten & $\begin{array}{l}\text { (Tuzlacı \& Sadıkoğlu 2007; } \\
\text { Kızılarslan 2008) }\end{array}$ \\
\hline Rumex acetosella L. & Polygonaceae & Kuzukulağı & $\begin{array}{l}\text { Aerial parts, } \\
\text { leaves } \\
\end{array}$ & The plant is eaten raw. & $\begin{array}{l}\text { Polat et al. 2015; Polat et al. 2013; } \\
\text { Polat \& Çakıroğlu 2018; Güneş 2010) }\end{array}$ \\
\hline Rumex crispus L. & Polygonaceae & $\begin{array}{l}\text { Ekşimek, } \\
\text { Labada }\end{array}$ & Leaves & The plant is eaten raw. & (Güneş 2010) \\
\hline Rumex tuberosus L. & Polygonaceae & Kislek & Stem, leaves & $\begin{array}{l}\text { Drink one tea glass of the } \\
\text { plant before the meal }\end{array}$ & $\begin{array}{l}\text { (Uysal et al. 2010; Polat et al. 2013; } \\
\text { Polat \& Çakıroğlu 2018; Onar 2006) }\end{array}$ \\
\hline $\begin{array}{l}\text { Rumex tuberosus L. subsp. } \\
\text { horizontalis (Koch) Rech. }\end{array}$ & Polygonaceae & $\begin{array}{l}\text { Trisog, Evelik, yumru } \\
\text { köklü kuzukulağı }\end{array}$ & Stem, leaves & Inf. & (Öztürk et al. 2013) \\
\hline
\end{tabular}


International Journal of Scientific and Technological Research

ISSN 2422-8702 (Online), DOI: 10.7176/JSTR/6-03-11

Special Issue of Health Sciences, Vol.6, No.3, 2020

\begin{tabular}{|c|c|c|c|c|c|}
\hline Botanical name & Family & Local name & $\begin{array}{l}\text { Plant part } \\
\text { used }\end{array}$ & $\begin{array}{l}\text { Preparation, } \\
\text { administration and use }\end{array}$ & References \\
\hline Rumex scutatus L. & Polygonaceae & $\begin{array}{l}\text { Kuzukulağı, } \\
\text { Tırşok }\end{array}$ & $\begin{array}{l}\text { Leaves, } \\
\text { aerial parts }\end{array}$ & $\begin{array}{l}\text { The plant is eaten raw., } \\
\text { Dec. }\end{array}$ & $\begin{array}{l}\text { (Tetik et al. 2013; Bulut et al. 2016; } \\
\text { Tetik 2011; Özgen et al. 2012; Tuzlacı } \\
\text { 2016) }\end{array}$ \\
\hline Salvia sclarea L. & Lamiaceae & $\begin{array}{l}\text { Paskulak, } \\
\text { Adaçayı }\end{array}$ & $\begin{array}{l}\text { Flowering } \\
\text { branches }\end{array}$ & Dec. & (Korkmaz \& Karakuş 2015) \\
\hline Sambucus ebulus L. & Caprifoliaceae & Sultan otu & Leaves & Dec. & $\begin{array}{l}\text { (Uysal et al. 2010; KızIlarslan 2008; } \\
\text { Onar 2006) }\end{array}$ \\
\hline Satureja cuneifolia Ten. & Lamiaceae & Kekik & Aerial parts & $\begin{array}{l}\text { Dec., } \\
\text { Inf. }\end{array}$ & (Oral 2007) \\
\hline Satureja hortensis L. & Lamiaceae & Kekik & Leaves & $\begin{array}{l}\text { Inf., Drink one cup of the } \\
\text { plant on an empty } \\
\text { stomach in the morning }\end{array}$ & $\begin{array}{l}\text { (Hayta 2014; Polat et al. 2013; } \\
\text { Tuzlacl 2016) }\end{array}$ \\
\hline $\begin{array}{l}\text { Satureja spicigera (C. Koch) } \\
\text { Boiss. }\end{array}$ & Lamiaceae & Kekik, zımpara & Leaves & Inf. & (Polat et al. 2015) \\
\hline Sideritis lanata L. & Lamiaceae & Karabaş otu & Aerial parts & Inf. & (Şenkardeş 2014) \\
\hline Sorbus aria L. & Rosaceae & Kuş üzümü & Fruits & O.Ad. & (Akgül et al. 2016) \\
\hline Sorbus aucuparia L. & Rosaceae & Üvez elması, Alıç & Fruits & Fresh O. Ad. eaten & Polat \& Satıl 2011 \\
\hline $\begin{array}{l}\text { Stachys cretica L. subsp. } \\
\text { mersinaea (Boiss.) Rech }\end{array}$ & Lamiaceae & \begin{tabular}{|l|} 
Boncuk şabla \\
deli çayı, rize çayı
\end{tabular} & Aerial part & Inf., Int & $\begin{array}{l}\text { (Özdemir \& Alpınar 2015; Tuzlacı } \\
\text { 2016) }\end{array}$ \\
\hline Styrax officinalis L. & Styracaceae & Günlük & Seeds & Seeds eaten raw & (Güneş et al. 2017; Güneş 2010) \\
\hline Taraxacum hybernum Stev. & Asteraceae & Karahindiba & $\begin{array}{l}\text { Flowers, } \\
\text { leaves }\end{array}$ & The plant is eaten raw & (Tetik et al. 2013; Tetik 2011) \\
\hline $\begin{array}{l}\text { Teucrium parviflorum } \\
\text { Schreber }\end{array}$ & Lamiaceae & Dağ kekiği & Aerial parts & Dec. & (Doğan 2014) \\
\hline Teucrium polium L. & Lamiaceae & Acı yavşan & Aerial parts & $\begin{array}{l}\text { Daily 1-2 cups of infusion } \\
\text { are drunk as tea. }\end{array}$ & $\begin{array}{l}\text { (Akaydın et al. 2013; Sargın 2015; } \\
\text { Tetik et al. 2013; Mükemre et al. } \\
\text { 2015; Polat \& Çakıroğlu 2018; } \\
\text { Karagöz 2013; Tetik 2011; Tuzlacı } \\
\text { 2016) }\end{array}$ \\
\hline Thymus fallax Fisch. \& Mey. & Lamiaceae & Kekik & Aerial parts & Inf., Dec., Int. & $\begin{array}{l}\text { (Altundağ \& Ozturk 2011; Altundağ } \\
\text { 2009) }\end{array}$ \\
\hline $\begin{array}{l}\text { Thymus kotschyanus Boiss. \& } \\
\text { Hohen. subsp. glabrescens } \\
\text { Boiss. }\end{array}$ & Lamiaceae & Kekik, Catri & Aerial parts & Inf., Dec., Int. & $\begin{array}{l}\text { (Altundağ \& Ozturk 2011; Mükemre et } \\
\text { al. 2015; Altundağ 2009) }\end{array}$ \\
\hline $\begin{array}{l}\text { Thymus longicaulis C. Presl. } \\
\text { subsp. longicaulis }\end{array}$ & Lamiaceae & Kekik & Aerial parts & $\begin{array}{l}\text { Inf., a glass per day for } 1 \\
\text { week }\end{array}$ & $\begin{array}{l}\text { (Kızılarslan 2008; Kartal \& Güneş } \\
\text { 2017) }\end{array}$ \\
\hline $\begin{array}{l}\text { Thymus migricus Klokov \& } \\
\text { Des.-Shost. }\end{array}$ & Lamiaceae & Kekik & Aerial parts & Inf., Dec., Int. & $\begin{array}{l}\text { (Altundağ \& Ozturk 2011; Altundağ } \\
\text { 2009) }\end{array}$ \\
\hline $\begin{array}{l}\text { Thymus praecox Opiz. subsp. } \\
\text { grossheimii (Ronniger) Jalas } \\
\text { var. grossheimii }\end{array}$ & Lamiaceae & Kekik & Aerial parts & Inf., Dec., Int. & $\begin{array}{l}\text { (Altundağ \& Ozturk 2011; Altundağ } \\
\text { 2009) }\end{array}$ \\
\hline $\begin{array}{l}\text { Thymus transcaucasicus } \\
\text { Ronniger }\end{array}$ & Lamiaceae & Kek otu, catıra & Whole plant & Inf. & $\begin{array}{l}\text { (Altundağ \& Ozturk 2011; Altundağ } \\
\text { 2009; Güneş \& Özhatay 2011; } \\
\text { Tuzlacı 2016) }\end{array}$ \\
\hline $\begin{array}{l}\text { Thymus sipyleus Boiss.subsp. } \\
\text { rosulans (Borbás) Jalas }\end{array}$ & Lamiaceae & Kekik & Aerial parts & $\begin{array}{l}\text { Inf., } \\
\text { Int. }\end{array}$ & (Tuzlacı \& Şenkardeş 2011;) \\
\hline $\begin{array}{l}\text { Thymus zygioides Griseb. var. } \\
\text { lycaonicus (Celak.) Ronniger }\end{array}$ & Lamiaceae & Kekik & Aerial parts & Inf. & (Deniz 2008; Tuzlacı 2016) \\
\hline Tribulus terrestris $\mathrm{L}$. & Zygophyllaceae & Demirci dikeni & Aerial parts & $\begin{array}{l}\text { Dec., drink one cup of the } \\
\text { plant on an empty } \\
\text { stomach in the morning }\end{array}$ & $\begin{array}{l}\text { (Hayta 2014; Onar 2006; Tuzlacı } \\
\text { 2016) }\end{array}$ \\
\hline $\begin{array}{l}\text { Tripleuospermum oreades } \\
\text { (Boiss.) Rech var. oreades }\end{array}$ & Asteraceae & \begin{tabular}{|l|} 
Papatya, \\
Oşoş \\
\end{tabular} & Aerial parts & Dec. & (Özgen et al. 2012) \\
\hline Urtica dioica L. & Urticaceae & $\begin{array}{l}\text { Isırgan, Isırganotu, } \\
\text { büyük Isırgan }\end{array}$ & Aerial parts & Dec., O.Ad. & $\begin{array}{l}\text { (Altundağ \& Ozturk 2011; Kılıç \& } \\
\text { Bağcı 2013; Uzun \& Kaya 2016; } \\
\text { Ozzhatay et al. 2006; Güneş 2010; } \\
\text { Altundağ 2009; Tuzlacı et al. 2010; } \\
\text { Özgen et al. 2012; Mumcu 2010; } \\
\text { Kültür 2007; } \\
\text { Tuzlacı 2016) }\end{array}$ \\
\hline
\end{tabular}


International Journal of Scientific and Technological Research

www.iiste.org

ISSN 2422-8702 (Online), DOI: 10.7176/JSTR/6-03-11

Special Issue of Health Sciences, Vol.6, No.3, 2020

\begin{tabular}{|c|c|c|c|c|c|}
\hline Botanical name & Family & Local name & $\begin{array}{l}\text { Plant part } \\
\text { used }\end{array}$ & \begin{tabular}{|l|} 
Preparation, \\
administration and use
\end{tabular} & References \\
\hline Valeriana dioscoridis Sm. & Valerianaceae & $\begin{array}{l}\text { Pisi otu, Pisik otu, Kedi } \\
\text { otu }\end{array}$ & Flowers & Inf. & (Demirci 2010; Tuzlacı 2016) \\
\hline Vicia ervilia Rafin L. & Fabaceae & Burçak & Seeds & Powdered, Int. & (Uğurlu et al. 2008) \\
\hline $\begin{array}{l}\text { Viscum album L. subsp. } \\
\text { abietis (Wiesb.) Abromeit. }\end{array}$ & Loranthaceae & Ökse otu & Fruits & \begin{tabular}{|l|} 
Int. Capsicum plaster, \\
(Fr) ext., Drink one glass \\
a day for $3-5$ weeks \\
\end{tabular} & $\begin{array}{l}\text { (Özdemir \& Alpınar 2015; Sargın et } \\
\text { al. 2015; Güneş et al. 2017; Güneş } \\
\text { 2010; Tuzlacı 2016) }\end{array}$ \\
\hline $\begin{array}{l}\text { Viscum album L. subsp. } \\
\text { album }\end{array}$ & Loranthaceae & Ökseotu, burç, çekim & $\begin{array}{l}\text { Fruits, leaves, } \\
\text { branches }\end{array}$ & $\begin{array}{l}\text { O.Ad., drink one teacup } \\
\text { two times a day for } 5-9 \\
\text { days; } \\
\text { Dec., Int. }\end{array}$ & $\begin{array}{l}\text { (Tetik et al. 2013; Cakilcioglu et al. } \\
\text { 2011; Fakir et al. 2009; Sağıroğlu et } \\
\text { al. 2013; Kızılarslan \& Özhatay 2012; } \\
\text { Özdemir \& Alpınar 2015; Ezer \& } \\
\text { Arısan 2006; Oral 2007; Doğan 2014; } \\
\text { Kızılarslan 2008; Onar 2006; Öztürk } \\
\text { 2006; Akan 2015; Genç 2003; Kültür } \\
\text { 2007; Genç 2006; Tuzlacı 2016) }\end{array}$ \\
\hline $\begin{array}{l}\text { Viscum album L. subsp. } \\
\text { austriacum (Wiesb.) Vollman }\end{array}$ & Loranthaceae & Çampurçu, Öksüotu & Leaves & \begin{tabular}{|l|} 
Leaves are cut into \\
pieces. Daily $1-2$ cups of \\
decoction are drunk.
\end{tabular} & $\begin{array}{l}\text { (Ugulu et al. 2009; Sargın et al. 2013; } \\
\text { Ezer \& Arısan 2006; Deniz 2008; } \\
\text { Ozkan \& Koyuncu 2005; Yeşilyurt } \\
\text { 2017) }\end{array}$ \\
\hline Zea mays $\mathrm{L}$. & Poaceae & MIsır & Stylus & $\begin{array}{l}\text { Drink one tea glass of the } \\
\text { plant three times a day }\end{array}$ & (Hayta 2014) \\
\hline $\begin{array}{l}\text { Ziziphora taurica Bieb. subsp. } \\
\text { taurica }\end{array}$ & Lamiaceae & Merze & Aerial parts & Inf., Int. & $\begin{array}{l}\text { (Altundağ \& Ozturk 2011; Altundağ } \\
\text { 2009; Tuzlacı 2016) }\end{array}$ \\
\hline Zizyphus jujuba Miller & Rhamnaceae & $\begin{array}{l}\text { Hırnap, } \\
\text { Hünnap }\end{array}$ & Fruits & Dec. & (Güneş 2010; Tuzlacı 2016) \\
\hline
\end{tabular}

O.Ad., Oral administration; Ext., External use; Int., Internal use; Inf., Infusion; Dec., Decoction;

\section{Materials and Methods}

This study is prepared by searching ethnobotanical studies accomplished in Turkey and by searching MSc and PhD theses at the Higher Education Council (Theses Center), with selecting plants used against hypertension. Also local plant names checked from different important sources in Turkish folk medicine (Tuzlac1 2016; Baytop 1994, Güner et al. 2012). Current plant names and families checked from www.plantlist.com.

In this study 150 ethnobotanical articles and some phytochemical articles were compiled. In 106 different records, data for the using medicinal plants against hypertension were founded and their used parts, uses were explained in details in Table 1. Mostly used plant families and plant parts showed in diagrams (Fig. 1-2).

\section{Results and Discussion}

This study, prepared by screening of ethnobotanical researches, that revealed 157 plant taxa, which were used for treatment of hypertension. These plant species are mostly from families Rosaceae, Lamiaceae, Asteraceae, Apiaceae, Polygonaceae, Loranthaceae and Rhamnaceae (Figure 1). Respectively mainly used taxa are Allium sativum L. (in 23 studies), Crataegus monogyna Jacq. subsp. monogyna (in 19 studies), Viscum album L. subsp. album (in 17 studies), Urtica dioica L. (in 11 studies), Olea europaea L. var. europaea, (in 10 studies), Punica granatum L. (in 9 studies), Lavandula stoechas L. subsp. stoechas (in 8 studies), Rosa canina L. (in 8 studies), Teucrium polium L. (in 8 studies), Petroselinum crispum (Miller) A. W. Hill. (in 7 studies) and Rosmarinus officinalis L. (in 7 studies). Mainly used plant parts are; leaves: $\% 32$, fruits: $\% 27$, flowers: $\% 16$, seeds: $\% 10$, root: $\% 9$ bulb: $\% 3$ and stem: $\% 3$ (Figure 2).

Clinical studies supports the antihypertensive effects of garlic (Allium sativum L.) (Ashraf et al. 2013), hawthorn (Crataegus sp.) (Chang et al. 2012), mistletoe (Viscum album L.) (Poruthukaren et al. 2014), stinging nettle (Urtica dioica L.) (Qayyum er al. 2016) and olive (Somova et al. 2003). For example in leaves of Crataegus monogyna and Crataegus laevigata the major constituents are flavonoids (hyperoside, rutin, quercetine, vitexin, vitexin- $\leq \leq$ rhamnoside, acetylvitexin- $2 \leq$ rhamnoside) and related proanthocyanidins. In the inflorescence, flavonol glycosides, mainly in the form of hyperoside, spiraeoside and rutin, are present. Hawthorn leaf with flower have positive inotropic, positive chronotropic and dromotropic; negative bathmotropic effect and increases coronary blood flow (Demirezer et al 2017). 


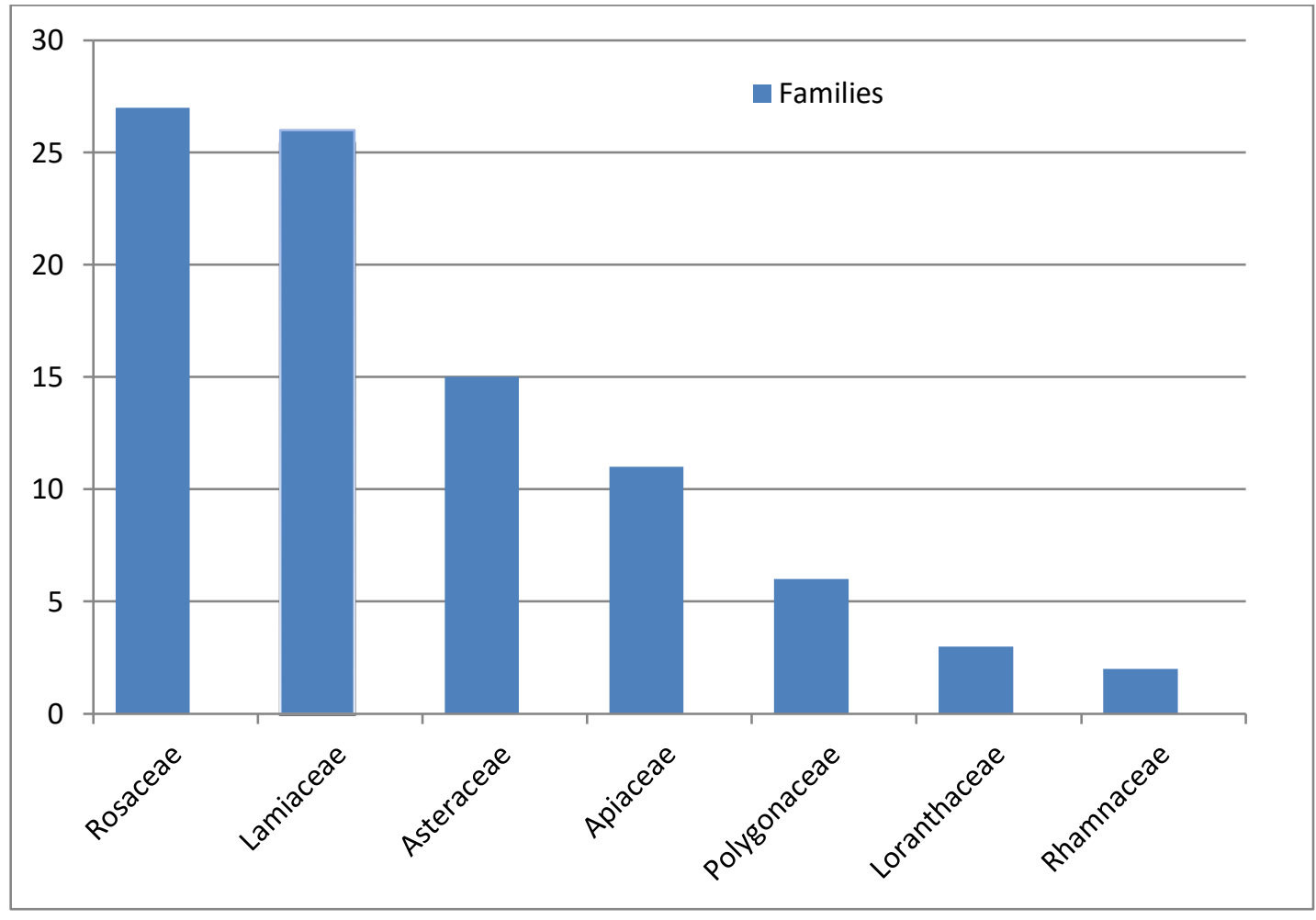

Fig. 1- The plant families used in traditional treatment of hypertension in Turkey

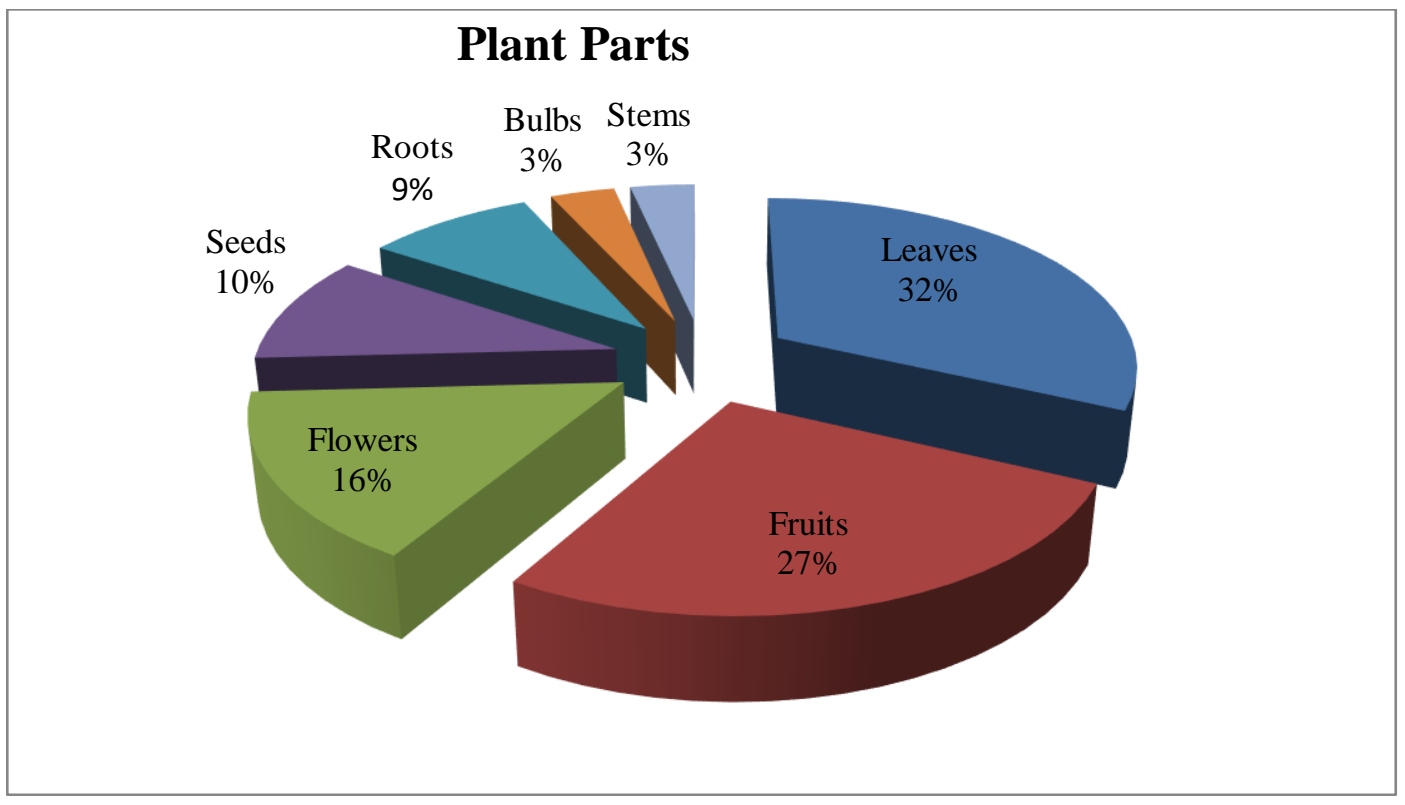

Fig. 2- The plant parts used in traditional treatment of hypertension in Turkey

We hope this study, which contains medicinal plants traditionally used in the treatment of hypertension in Turkey, will contribute to the development of conventional drugs originate from plant sources, that can be useful in the treatment of hypertension. 


\section{Acknowledgement}

This study is founded by Istanbul University Scientific Research Projects Department with 39753 coded project.

\section{References:}

Akan, H., \& Sade, Y.B., (2015). Kahta (Adiyaman) merkez ve Narince Köyü’nün etnobotanik açıdan araştırılması. BË̈ Fen Bil Derg. 4(2), 219-248.

Akan, H., Balos, M.M., \& Tel, Z.A. (2013). The Ethnobotany of some Legume plants around Birecik (Şanliurfa). The Journal of Adyutayam, 1 (1), 31-39.

Akaydin, G., Şimşek, I., Arituluk, Z.C., \& Yeşilada, E. (2013). An ethnobotanical survey in selected towns of the Mediterranean subregion (Turkey). Turk J Biol, 37, 230-247.

Akbulut, S., \& Bayramoglu, M.M., (2013). The Trade and Use of Some Medical and Aromatic Herbs in Turkey. Studies on Ethno-Medicine, 7(2), 67-77.

Akgul, A., Serdar, G.S., Yıldırım, S., Seçmen, O., \& Doğan, Y., (2018). An ethnobotanical study in Midyat (Turkey), a city on the silk road where cultures meet. J Ethnobiol Ethnomed, 14(12), 118.

Akgül, G., Yılmaz, N., Celep, A., Celep, F., \& Çakılcığlu, U. (2016). Ethnobotanical purposes of plants sold by herbalists and folk bazaars in the center of Cappadocica (Nevşehir, Turkey), Indian J Tradit Know, 15(1), 103-108.

Alpaslan, Z. (2012). The ethnobotanical properties of Ergan mountain (Erzincan). MSc Thesis, Erzincan University, Erzincan.

Altundağ, E., \& Ozturk, M. (2011). Ethnomedicinal studies on the plant resources of east Anatolia. Procedia-Social and Behavioral Sciences, 19, 756-777.

Altundağ, E. (2009). Iğdır ilinin (Doğu Anadolu Bölgesi) doğal bitkilerinin halk tarafından kullanımı. PhD Thesis, Istanbul University, Istanbul.

Arı, S., Temel, M., Kargığlu, M., \& Konuk, M., (2015). Ethnobotanical survey of plants used in Afyonkarahisar- Turkey. J Ethnobiol Ethnomed, 11, 84.

Ashraf, R., Khan, R.A., Ashraf, I., \& Qureshi, A.A. (2013). Effects of Allium sativum (Garlic) on systolic and diastolic blood pressure in patients with essential hypertension. Pak J Pharm Sci, 26 (5), 859-863.

Baytop, T. (1994). Türkçe Bitki Adları Sözlüğü, Ankara: Türk Dil Kurumu Yayınları: 578.

Bulut, G., \& Tuzlac1, E. (2013). An ethnobotanical study of medicinal plants in Turgutlu (Manisa Turkey). J Ethnopharmacol, 149, 633-647.

Bulut, G., Biçer, M., \& Tuzlacı, E. (2016). The folk medicinal plants of Yüksekova (HakkariTurkey). J Fac Pharm, 46(2), 115-124.

Bulut, G., Haznedaroğlu, M.Z., Doğan, A., Koyu, H., \& Tuzlacı, E. (2017). An ethnobotanical study of medicinal plants in Acipayam (Denizli-Turkey). J Herb Med, 10, 64-81.

Bulut, G., Tuzlac1, E., Doğan, A., \& Şenkardeş, İ. (2014). An ethnopharmacological review on the Turkish Apiaceae species. J Fac Pharm, 44 (2), 163-179. 
Bulut, G.E. (2008). Ethnobotanical investigations in Bayramiç (Çanakkale), PhD Thesis, Marmara University, Istanbul.

Cakilcioglu, U., \& Turkoglu, I., (2010). An ethnobotanical survey of medicinal plants in Sivrice (Elazig-Turkey) J Ethnopharmacol. 132, 165-175.

Cakilcioglu, U., Khatun, S., Turkoglu, I., \& Hayta, S., (2011). Ethnopharmacological survey of medicinal plants in Maden (Elazig-Turkey). J Ethnopharmacol, 137 (1), 469-486.

Chang, Q., Zuo, Z., Harrison, F., \& Chow, M.S.S., (2002). Hawthorn. J Clin Pharmacol, 42, 605612.

Çakılcıŏglu, U., Şengün, M.T., \& Türkoğlu, İ., (2010). An ethnobotanical survey of medicinal plants of Yazıkonak and Yurtbaşı Districts of Elazı̆g Province, Turkey. J Med Plant Res, 4 (7), 567-572.

Demirci, S., \& Özhatay, N. (2012). An ethnobotanical study in Kahramanmaraş (Turkey); wild plants used for medicinal purpose in Andirin, Kahramanmaraş. Turk J Pharm Sci, 9 (1), 75-92.

Demirci, S. (2010). Ethnobotanical study in Andırın (Kahramanmaraş) district, MSc Thesis, İstanbul University, İstanbul.

Demirezer, Ö. et al. (2017). FFD Monografları Bitkiler ve Etkileri, In: Crataegus laevigataCrataegus monogyna, edited by Şöhretoğlu D, Akademisyen Kitabevi, Ankara, 297-308.

Deniz, L. (2008). The flora and its ethnobotanic evaluation of Usak University 1 Eylül Campus (Usak), Msc Thesis, Afyon Kocatepe University, Afyon.

Doğan, A. (2014). (Tunceli) yöresinde etnobotanik araştırmalar, PhD Thesis, Marmara University, İstanbul.

Ekşi, G. (2012). Türkiye'de Allium L. cinsine ait (Sect. Allium) endemik türler üzerinde morfolojik ve etnobotanik bir çalışma, $M S c$ Thesis, Ankara Üniversitesi, Ankara.

Erdoğan, R. (2011). Ethnobotanical features some of the wild on the Sarıveliler (Karaman) and its environment ethnobotanic, MSc Thesis, Selçuk University, Konya.

Eşen, B. (2008). The ethnobotanical properties of Aydınlar village and its vicinity (Erdemli/ Mersin), MSc Thesis, Selçuk University, Konya.

Ezer, N., \& Arısan, MÖ (2006). Folk Medicines in Merzifon (Amasya, Turkey). Turk J Bot, 30: 223 230.

Fakir, H., Korkmaz, M., \& Güller, B. (2009). Medicinal Plant Diversity of Western Mediterrenean Region in Turkey, J Appl Biol Sci, 3 (2), 30-40.

Genç, GE., \& Özhatay, N. (2006). An ethnobotanical study in Çatalca (European part of Istanbul) II. Turk J Pharm Sci, 3 (2), 73-89.

Genç, GE. (2003). Çatalca yöresinde etnobotanik bir araştırma. MSc Thesis, İstanbul Üniversitesi, İstanbul)

Gençay, A. (2007). Ethnobotanical aspects of Cizre (Şırnak), MSc Thesis, Yüzüncü Yıll University, Van.

Güldaş, N. (2009). The investigation usage of some ethnobotanical valued plants in Adıyaman, $M S c$ Thesis, Firat University, Elazığ. 
Güler, B., Manav, E., \& Uğurlu, E., (2015). Medicinal plants used by traditional healers in Bozüyük (Bilecik- Turkey), J Ethnopharmacol, 173, 39-47.

Güner, A. et al. (2012). Türkiye Bitkileri Listesi (Damarlı Bitkiler), Nezahat Gökyiğit Botanik Bahçesi ve Flora Araştırmaları Derneği Yayını, İstanbul.

Güner, A., Özhatay, N., Ekim, T., \& Başer, KHC. (2000). Flora of Turkey and the East Aegean Islands, vol 11, Edinburgh University Press, Edinburgh.

Güneş, F., \& Özhatay, N. (2011). An ethnobotanical study from Kars (Eastern) Turkey. Biodicon, 4 (1), 30- 41.

Güneş, S. (2010). Karaisalı (Adana) and villages natural plants used by the public, ethnobotanical respect investigation, $M S c$ Thesis, Niğde University, Niğde.

Güneş, S., Savran, A., Paksoy, MY., Koşar, M., \& Çakılcıŏglu, U. (2017). Ethnopharmacological survey of medicinal plants in Karaisalı and its surrounding (Adana-Turkey), J Herb Med, 8, 6875 .

Gürdal, B., \& Kültür, Ş. (2013). An ethnobotanical study of medicinal plants in Marmaris (Mugla, Turkey). J Ethnopharmacol. 146, 113-126.

Güzel, Y., Güzelşemme, M., \& Miski, M. (2015). Ethnobotany of medicinal plants used in Antakya: A multicultural district in Hatay Province of Turkey. J Ethnopharmacol, 174, 118-152.

Güzelşemme, M. (2014). Antakya'da kullanılan tıbbi bitkiler ile yabani gıda bitkileri. MSc Thesis, Mustafa Kemal University, Hatay.

Han, Mİ., \& Bulut, G. (2015). The folk-medicinal plants of Kadişehri (Yozgat - Turkey). Acta Soc Bot Pol, 84 (2), 237-248.

Hayta, S., Polat, R., \& Selvi, S. (2014). Traditional uses of medicinal plants in Elazığ (Turkey). J Ethnopharmacol, 154 (3), 613-623.

He, J., \& Whelton, PK. (1997). Epidemiology and prevention of hypertension. Med Clin North Am, 81, 7-1097.

Karagöz, KF. (2013). Suşehri (Sivas) bölgesinin etnobotanik açıdan değerlendirilmesi. MSc Thesis, Afyon Kocatepe Üniversitesi, Afyon.

Karaman, S., \& Kocabaş, ZY. (2001). Traditional medicinal plants of K. Maraş (Turkey). The Sciences, 1 (3), 125-128.

Kargıŏlu, M., Cenkci, S., Serteser, A., Konuk, M., \& Vural, G. (2010). Traditional Uses of Wild Plants in the Middle Aegean Region of Turkey. Hum Ecol. 38, 429-450.

Kartal, Ç., \& Güneş, F. (2017). Medicinal Plants Used in Meriç Town from Turkey. Indian J Pharm Educ, 51(3), 249-53.

Kaval, I., Behçet, L., \& Çakılcığlu, U. (2014). Ethnobotanical study on medicinal plants in Geçitli and its surrounding (Hakkari- Turkey). J Ethnopharmacol, 155, 171-184.

Kılıç, O., \& Bağc1, E. (2013). An ethnobotanical survey of some medicinal plants in Keban (Elazı̆̆Turkey), J Med Plant Res, 7 (23), 1675-1684.

Kızılarslan, Ç., \& Özhatay, N. (2012). Wild plants used as medicinal purpose in the south part of İzmit (Northwest Turkey). Turk J Pharm Sci, 9 (2), 199-218. 
Kızlarslan, Ç. (2008). An ethnobotanical survey in the South part of İzmit Gulf. MSc Thesis, İstanbul University, İstanbul.

Koçyiğit, M., \& Özhatay, N. (2006). Wild plants used as medicinal purpose in Yalova (North Turkey). Turkish J Pharm Sci, 3 (2), 91-103.

Korkmaz, M., \& Karakuş, S. (2015). Traditional uses of medicinal plants of Üzümlü District, Erzincan, Turkey. Pak. J. Bot, 47 (1), 125-134.

Kültür, Ş. (2007). Medicinal plants used in Kırklareli province (Turkey). J Ethnopharmacol, 111 (2), 341-64.

Metin, A. (2009). Ethnobotanical features of plants in Mut (Mersin) and its environments. Msc Thesis, Selçuk University, Konya.

Mumcu, AÖ. (2010). Işsk Dağı ve çevresinde yetişen bitkiler üzerinde farmasötik botanik yönünden araştırmalar. PhD Thesis, Ankara University, Ankara.

Mükemre, M., Behçet, L., \& Çakıılcıŏlu, U. (2015). Ethnobotanical study on medicinal plants in villages of Çatak (Van-Turkey). J Ethnopharmacol, 166, 361-374.

Onar, S. (2006). Bandırma (A1 (A), Balıkesir) ve çevresinin etnobotaniği. MSc Thesis, Çanakkale Onsekiz Mart Üniversitesi, Çanakkale.

Oral, ÇD. (2007). Ethnobotanical studies on folk medicines used in Konya. Msc Thesis, Gazi University, Ankara.

Ozkan, AMG., \& Koyuncu, M. (2005). Traditional medicinal plants used in Pınarbaşı area (KayseriTurkey). Turkish J Pharm Sci, 2 (2), 63-82.

Özdemir, E., \& Alpınar, K. (2015). An ethnobotanical survey of medicinal plants in western part of central Taurus Mountains: Aladaglar (Nigde-Turkey). J Ethnopharmacol, 166, 53-65.

Özgen, U., Kaya, Y., \& Houghton, P. (2012). Folk medicines in the villages of Ilıca district (Erzurum, Turkey). Turk J Biol, 36, 93- 106.

Özhatay, N., Akalın, E., Genç, EG., \& Kültür, Ş. (2006). Ethnomedicinal uses of the wild vascular plants from European Turkey (Turkish Thrace). In: Plant, fungal and habitat diversity investigation and conservation. Proceedings of IV. Balkan Botanical Congress Sofia 20-26th June, 2006, Institute of Botany, Bulgarian Academy of Sciences. Sofia, Bulgaria, 613-623.

Özhatay, N., Kültür, Ş., \& Gürdal, B. (2013). Check-list of additional taxa to the supplement flora of Turkey VI. J Fac Pharm, 43, 33-82.

Özhatay, N., Kültür, Ş., \& Gürdal, B. (2015). Check-list of additional taxa to the supplement flora of Turkey VII. J Fac Pharm, 45, 61-86.

Özhatay, N., Kültür, Ş., \& Gürdal, B. (2017). Check-list of additional taxa to the supplement flora of Turkey VIII. J Fac Pharm, 47 (1), 31-46.

Öztürk, M. (2006). The flora and ethnobotany of Nizip region (Aksaray). MSc Thesis, Selçuk University, Konya.

Öztürk, M., Uysal, I,. Gucel, S., Altundağ, E., Dogan, Y., \& Baslar, S. (2013). Medicinal uses of natural dye- yielding plants in Turkey. RJTA, 17, 69-80.

Polat, R., \& Çakılcıŏlu, U. (2018). Ethnobotanical study on medicinal plants in Bingöl (City center) (Turkey). J Herb Med, 01, 007. 
Polat, R., \& Sat1l, F. (2011). An ethnobotanical survey of medicinal plants in Edremit Gulf (Balıkesir - Turkey). J Ethnopharmacol, 139 (2), 626-641.

Polat, R., Cakilcioglu, U., \& Satıl, F. (2013). Traditional uses of medicinal plants in Solhan (BingölTurkey). J Ethnopharmacol, 148 (3), 951-963.

Polat, R., Çakılcığlu, U., Kaltalioğlu, K, Ulusan MD., \& Tümen, Z. (2015). An ethnobotanical study on medicinal plants in Espiye and its surrounding (Giresun-Turkey). J Ethnopharmacol, 163, 111.

Polat, R., Çakılcığlu, U., Ulusan, MD., Gür, F., \& Tümen, Z. (2015). Investigations of ethnobotanical aspect of wild plants sold in Espiye (Giresun/Turkey) local markets. Biodicon, 8 (3), 114-119.

Polat, R. (2010). Havran ve Burhaniye (Balıkesir) çevresinde tarımsal biyoçeşitlilik ve etnobotanik araştırmaları. PhD Thesis, Balıkesir Üniversitesi, Balıkesir.

Poruthukaren, KJ., Palatty, PL., Baliga, MS., \& Suresh, S. (2014). Clinical Evaluation of Viscum album Mother Tincture as an Antihypertensive: A Pilot Study. J Evid Based Complementary Altern Med, 19 (1), 31-35.

Qayyum, R., et al. (2016). Mechanisms underlying the antihypertensive properties of Urtica dioica. J Transl Med, 14 (1), 254.

Saday, H. (2009). Ethnobotanical properties of Güzeloluk village and its vicinity. MSc Thesis, Selçuk University, Konya.

Sağıroğlu, M., Dalgıç, S., \& Toksoy, S. (2013). Medicinal plants used in Dalaman (Muğla), Turkey. J Med Plant Res, 7 (28), 2053-2066.

Saraç, DU. (2013). Ethnobotanic features of Rize province, MSc Thesis, Karadeniz Technical University, Trabzon).

Sargın, SA., Akçicek, E., \& Selvi, S. (2013). An ethnobotanical study of medicinal plants used by the local people of Alaşehir (Manisa) in Turkey. J Ethnopharmacol, 150 (3), 860-874.

Sargin, SA. (2015). Ethnobotanical survey of medicinal plants in Bozyazı district of Mersin, Turkey. J Ethnopharmacol, 173, 105-126.

Sargin, SA, Selvi, S., \& Büyükcengiz, M. (2015). Ethnomedicinal plants of Aydincık District of Mersin, Turkey, J Ethnopharmacol, 174, 200-216.

Savran, A., Bağc1, Y., \& Kargı̆ğlu, M. (2009). Vernacular names and ethnobotanical aspects of some species in Gemerek (Sivas) and its vicinity, AKU J Sci Eng, 8, 313- 21.

Sezik, E., Yeşilada, E., Honda, G., Takaishi, Y., Takeda, Y., \& Tanaka, T. (2001). Traditional medicine in Turkey X. Folk medicine in Central Anatolia. J Ethnopharmacol, 75, 95-115.

Simsek, I., Aytekin, F., Yesilada, E., \& Yildirimli, S. (2004). An Ethnobotanical Survey Of The Beypazar1, Ayas, And Güdül District Towns Of Ankara Province (Turkey). Economic Botany, 58(4), 705-720.

Somova, LI., Shode, FO., Ramnanan, P., \& Nadar A. (2003). Antihypertensive, antiatherosclerotic and antioxidant activity of triterpenoids isolated from Olea europaea subsp. africana leaves. $J$ Ethnopharmacol, 84, 299-305.

Şahin, YS. (2014). Medicinal plants sold in Gaziantep herbalists and their ethnobotanical aspects. MSc Thesis, Gaziantep University, Gaziantep. 
Şenkardeş, İ. (2014). Ethnobotanical investigations in southern districts (Acıgöl, Derinkuyu, Gülşehir, Nevşehir-Central district, Ürgüp) of Nevşehir. PhD Thesis, (Marmara University, Istanbul).

Tekin, S. (2011). Ethnobotanic aspects of Üzümlü (Erzincan) Town. MSc Thesis, Erzincan University, Erzincan.

Tetik, F. (2011). A research on the ethnobotanical valued plants in Malatya province. MSc Thesis, Firat University, Elazı $\breve{g})$,.

Tetik, F., Civelek, S., \& Cakilcioglu, U. (2013). Traditional uses of some medicinal plants in Malatya (Turkey). J Ethnopharmacol, 146 (1), 331-346.

Tuzlac1, E., \& Alparslan, DF. (2013). Turkish Folk Medicinal Plants, Part V: Babaeski (Kirklareli). J Fac Pharm, 39 (0), 11-23.

Tuzlacı, E., \& Doğan, A. (2010). Turkish folk medicinal plants, IX: Ovacık (Tunceli). Marmara Pharm J, 14,136-143.

Tuzlac1, E., \& Erol, MK. (1999). Turkish folk medicinal plants. Part II: Egirdir (Isparta), Fitoterapia, 70, 593-610.

Tuzlacı, E., \& Sadikoğlu, E. (2007). Turkish folk medicinal plants part VI: Koçarlı (Aydın). J Fac Pharm, 39, 25-37.

Tuzlacı, E., \& Şenkardeş, İ. (2011). Turkish folk medicinal plants part X: Ürgüp Nevşehir. Marmara Pharm J, 15, 58-68.

Tuzlacı, E., Tolon, E., (2000). Turkish folk medicinal plants. Part III: Şile (İstanbul). Fitoterapia, 71, 673-685.

Tuzlacı, E., İşbilen, DFA., \& Bulut, G. (2010). Turkish folk medicinal plants, Part VIII: Lalapaşa (Edirne). Marmara Pharm J, 14 47-52.

Tuzlacı, E. (2016). Türkiye Bitkileri Geleneksel İlaç Rehberi. Ístanbul Tıp Kitabevleri, İstanbul.

Tütenocaklı, T. (2014). Yenice (Çanakkale) ve çevresinde tarımsal bitki biyoçeşitliliği ve etnobotanik araştırmalar. PhD Thesis, Çanakkale Onsekiz Mart Üniversitesi, Çanakkale.

Ugulu, I., Baslar, S., Yorek, N., \& Doğan, Y. (2009). The investigation and quantitative ethnobotanical evaluation of medicinal plants used around Izmir province, Turkey. J Med Plants Res, 3(5) 345-367.

Uğurlu, E., \& Seçmen, O. (2008). Medicinal plants popularly used in the villages of Yunt Mountain (Manisa- Turkey). Fitoterapia, 79, 126-131.

Uysal, I. (2010). An overview of plant diversity of Kazdagi (Mt. Ida) Forest National Park Turkey. Journal of Environmental Biology, 31, 141-147.

Uysal, I., Gücel, S., Tütenocaklı, T., \& Öztürk, M. (2012). Studies on the medicinal plants of Ayvacık- Çanakkale In Turkey, Pak. J. Bot., 44, 239-244.

Uysal, İ., Onar, S., Karabacak, E., \& Çelik, S. (2010). Ethnobotanical aspects of Kapıdağ Peninsula (Turkey), Biodicon, 3(3), 15-22.

Uzun, M., \& Kaya, A. (2016). Ethnobotanical research of medicinal plants in Mihalgazi (Eskişehir, Turkey), Pharmaceutical Biology, 54 (12), 2922-2932. 
Vural, G. (2008). Ethnobotanical features some of the willd plants on the Honaz mountain and its environment ethnobotanic. MSc Thesis, Afyon Kocatepe University, Afyon.

Williams, B., Mancia, G., Spering, W., \& Agabiti, RE. (2018). The Task force for the management of arterial hypertension of the European Society of Cardiology (ESC) and the European Society of Hypertension (ESH). European Heart Journal, 00 1-98.

Yazıcıŏglu, A., \& Tuzlacı, E. (1995). Folk medicinal plants of Trabzon (Turkey). Marmara Pharm $J, 11(1-2)$ 333-342.

Yeşil, Y., \& Akalın, E. (2009). Folk medicinal plants in Kürecik area (Akçadağ/Malatya-Turkey), Turk J Pharm Sci, 6, 207-220.

Yeşilyurt, EB., Şimşek, I., Akaydın, G., \& Yeşilada, E. (2017). An ethnobotanical survey in selected districts of the Black Sea region (Turkey). Turk J Bot, 41, 47-62. 